\title{
Artroplastia estabilizadora y de interposición para artrodesis previa de la articulación trapecio-metacarpiana del pulgar
}

\author{
Dr. Antonio De Santolo R.(1), Dra. Magalli C. Ortiz ${ }^{(2)}$, \\ Dr. Leonardo E. Briceño(3), T. O. Nyoka Cevedo R. ${ }^{(4)}$ \\ ${ }^{(1)}$ Profesor titular Facultad de Medicina UCV y del Instituto Médico La Floresta. Caracas.

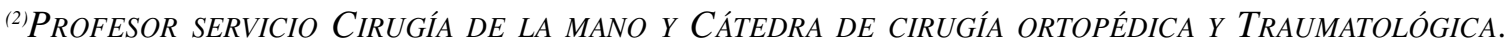 \\ H. U. C. E INSTITUTO MÉdico LA FLORESTA. CARACAS. \\ ${ }^{(3)}$ Cirujano de Mano Instituto Médico La Floresta. Caracas. \\ ${ }^{(4)}$ Fisioterapeuta de mano Y miembro superior. Instituto Médico La Floresta. Caracas.
}

La articulación carpometacarpiana o trapecio-metacarpiana del pulgar es la más importante para las diferentes pinzas. $\mathrm{Su}$ artrodesis fue realizada en el pasado frecuentemente, pero ella deja como residuo una severa incapacidad funcional. Con los avances actuales de los modernos procedimientos quirúrgicos, la artrodesis de la trapecio-metacarpiana estaría inclinada sólo en casos excepcionales. Presentamos el resultado de 8 pacientes con artrodesis de la trapecio-metacarpiana realizadas en otras instituciones, nosotros las convertimos en artroplastias estabilizadoras y de interposición con excelentes o buenos resultados, 5 de ellos fueron artrodesados por secuelas de fracturas y 3 por Rizoartrosis. La edad de los pacientes osciló entre 37 y 59 años con promedio de 43 , ninguno de los pacientes tenía un trabajo exigente o manual.

El programa de Rehabilitación es muy importante para obtener excelentes o buenos resultados, en 3 obtuvimos excelentes resultados, 2 buenos y 1 regular, según la escala de EATON, sólo con 3 complicaciones.

Son nuestras conclusiones que la conversión de una artrodesis en artroplastia en la base del pulgar es muy satisfactoria, el paciente lo agradece mucho porque le disminuye la incapacidad, le elimina el dolor y deja una trapecio-metacarpiana muy estable por la reconstrucción de su ligamento más importante, con unos de los tendones del Abductor largo del pulgar (digástrico), interponiendo alrededor de éste la cápsula articular.

Palabras clave: Artroplastia, artrodesis previa, articulación CMC pulgar.
Stabilization/interposition arthroplasty in previous arthrodesis of the carpusmetacarpal joint thumb. The trapezio-metacarpal joint of the Thumb it is the most important for the different pincers. This arthrodesis was frequently carried out in the past, but leaves as residual a severe functional impairment. In the current advances of the modern surgical procedures, the arthrodesis of the trapeziometacarpal joint would only be indicated in exceptional cases.

We present the result of 8 patients with arthodesis oh the trapezio-metacarpal joint carried out in other institutions, where we transform them into arthroplasty for interposition withgood results, five of them carried out arthrodesis for sequels of fractures, and three for Osteoarthritis - Rizoarthrosis. The age of the patients oscillated between 37 and 59 years, with average of 43 years, no patients had a demanding manual work.

The rehabilitation program is very important to obtain excellent results, we obteined 3 excellent results 2 good results and 1 fair; according to the scale or EATON; only 3 complications.

Our conclusions is that the conversion of an arthrodesis in arthroplasty in the base the thumb is very satisfactory, the patient thanks a lot, because dimishes the impairment, eliminates the pain and leaves a very stable trapezio-metacarpal joint the technical point of surgical important is the reconstruction of the beak ligament with one tendon of the APL and capsule around it.

Key words: Arthroplasty, arthrodesis, CMC joint thumb. 


\section{INTRODUCCIÓN}

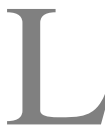

a articulación trapecio-metacarpiana es la más importante para las diferentes pinzas y función del pulgar (3). Su afectación más frecuente se produce: en artritis reumatoidea, post-traumática secuela de fracturas o en la osteoartrosis con presencia de rizoartrosis; como consecuencia de la afectación se presenta una sintomatología muy incapacitante, caracterizada por dolor, y en estados avanzados deformidad de la articulación trapecio-metacarpiana inclusive del pulgar, que dificulta hasta las actividades de la vida diaria.

En el pasado fue muy frecuente la artrodesis de la articulación trapecio-metacarpiana como secuela de las afecciones antes mencionadas, pero en los últimos 15 años los avances nos han permitido eliminar la incapacidad que esta artrodesis produce, ya que las artroplastias per-

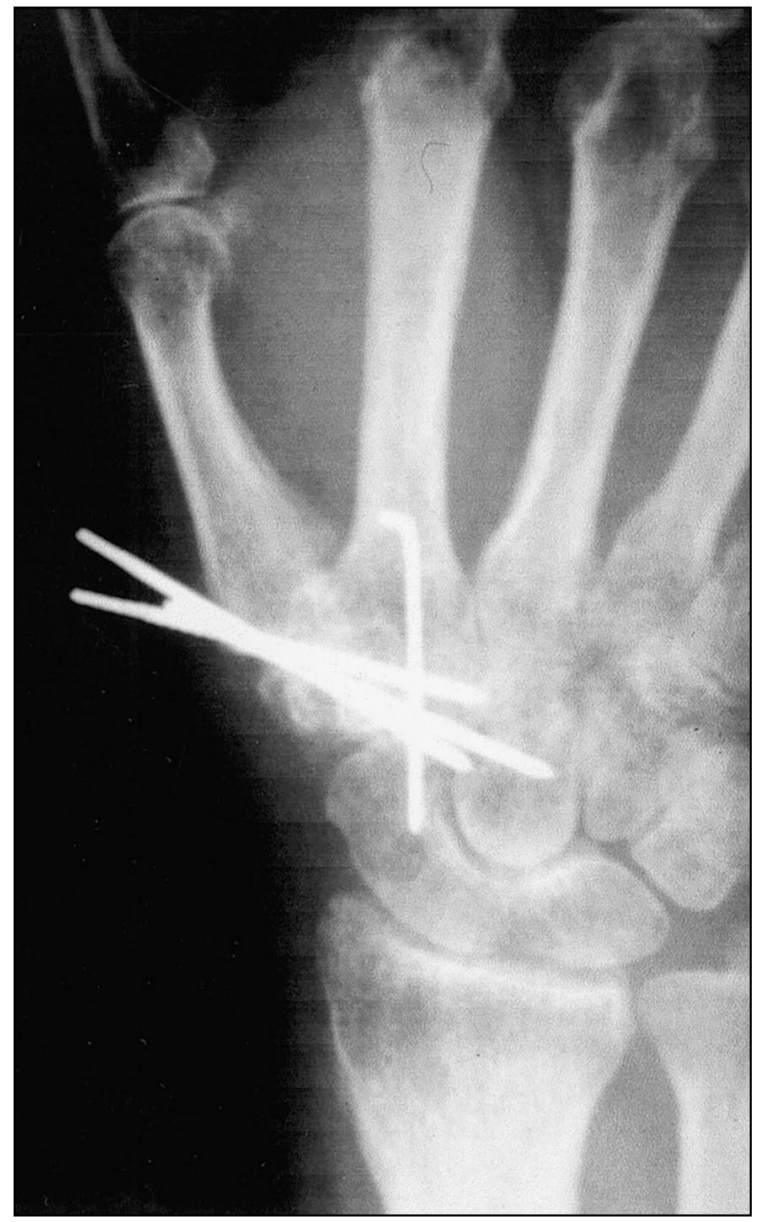

Figura 1. Artrodesis de la C.M.C. pulgar dominante, mujer de 58 años. miten mantener la movilidad del pulgar; dejando la artrodesis para condiciones especiales y casos excepcionales.

\section{RECUENTO ANATÓMICO}

La articulación carpo-metacarpiana del pulgar es una articulación en forma de silla de montar, incongruente, con una configuración que permite movimientos de flexión-extensión, abducción-addución, oposición y circunducción.

La estabilidad está dada por los ligamentos (2), y el más importante es el palmar oblicuo u oblicuo anterior, que se inserta en la parte palmar del metacarpiano. El primer compartimiento de los tendones extensores está directamente sobre la articulación radialmente, y la arteria radial recorre el dorso de la cápsula articular.

El nervio radial sensitivo en sus varias ramas terminales está inmediatamente en la zona por debajo del tejido subcutáneo adyacente a la ar-

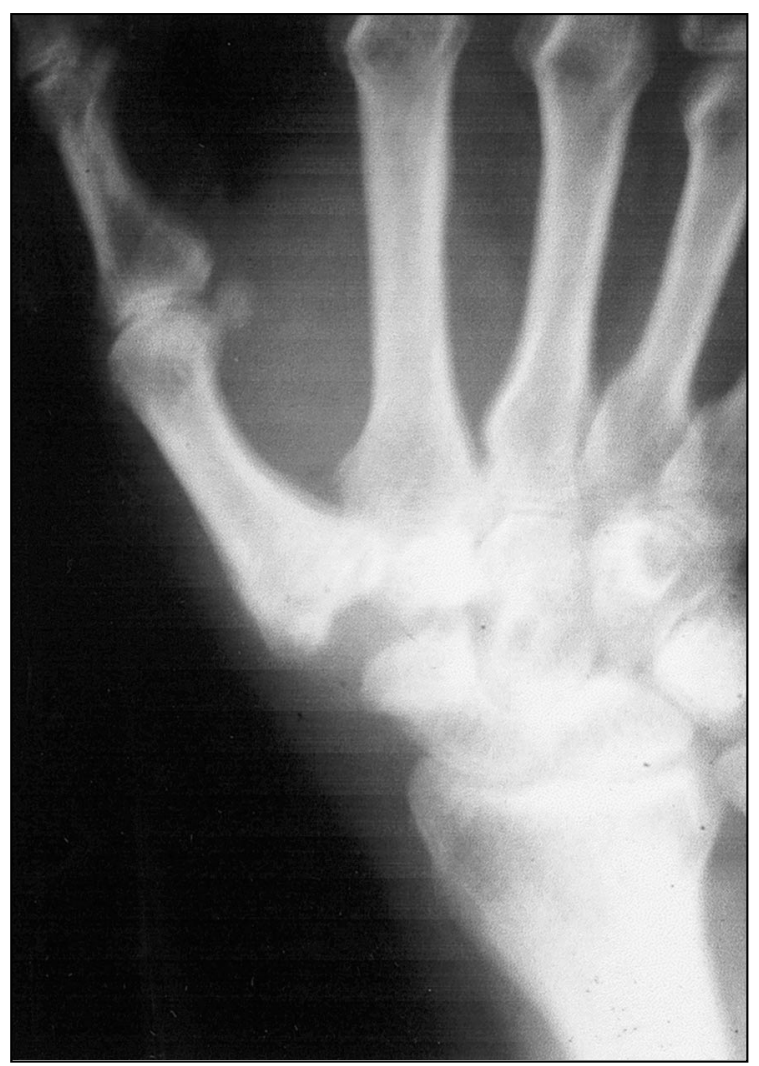

Figura 2. Artroplastia por interposición y estabilizadora. Resultado radiológico de figura 1. 


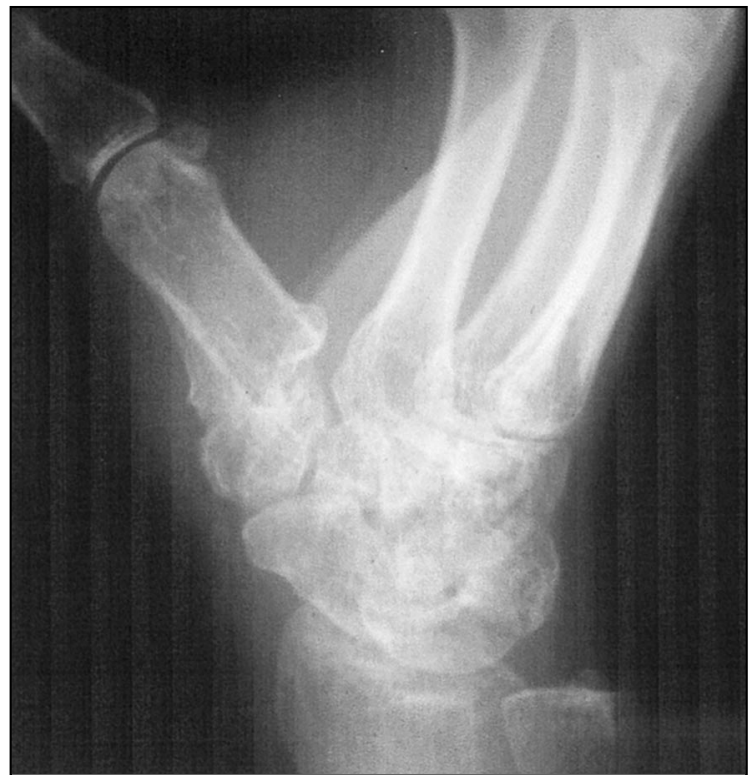

Figura 3. Artrodesis de la C.M.C. hombre de 39 años, incapacidad para oposición y de pinzas finas.

ticulación. Es la articulación más móvil del organismo, siendo muy importante para las funciones del pulgar.

\section{MATERIAL Y MÉTODO}

Durante 10 años, 1991-2000, realizamos 68 artroplasias por interposición en una sola de las instituciones donde ejercemos, intervenciones que comenzamos a realizarlas en 1887, de ellas, 8 pacientes habían sido artrodesados en su trapecio-metacarpianas en otras instituciones.

La edad de los pacientes osciló entre 31 a 59 años, promedio 43 años, 6 mujeres, 2 hombres, ninguno tenía un trabajo manual que exigiera máxima estabilidad y fuerza, pero a pesar de ello habían sido artrodesados: 5 pacientes por secuelas de fracturas articulares de la trapeciometacarpiana y 3 por rizoartrosis, ninguno tenía artritis reumatoidea.

La duración de la artrodesis antes de convertirlas a artroplastia fue de 1 a 4 años, y todos los pacientes refirieron además de dolor: incapacidad para la circunducción y oposición completa del pulgar, también el no poder aplanar la mano sobre una superficie por pérdida del arco transverso del carpo, y dificultad para agarrar objetos pequeños.

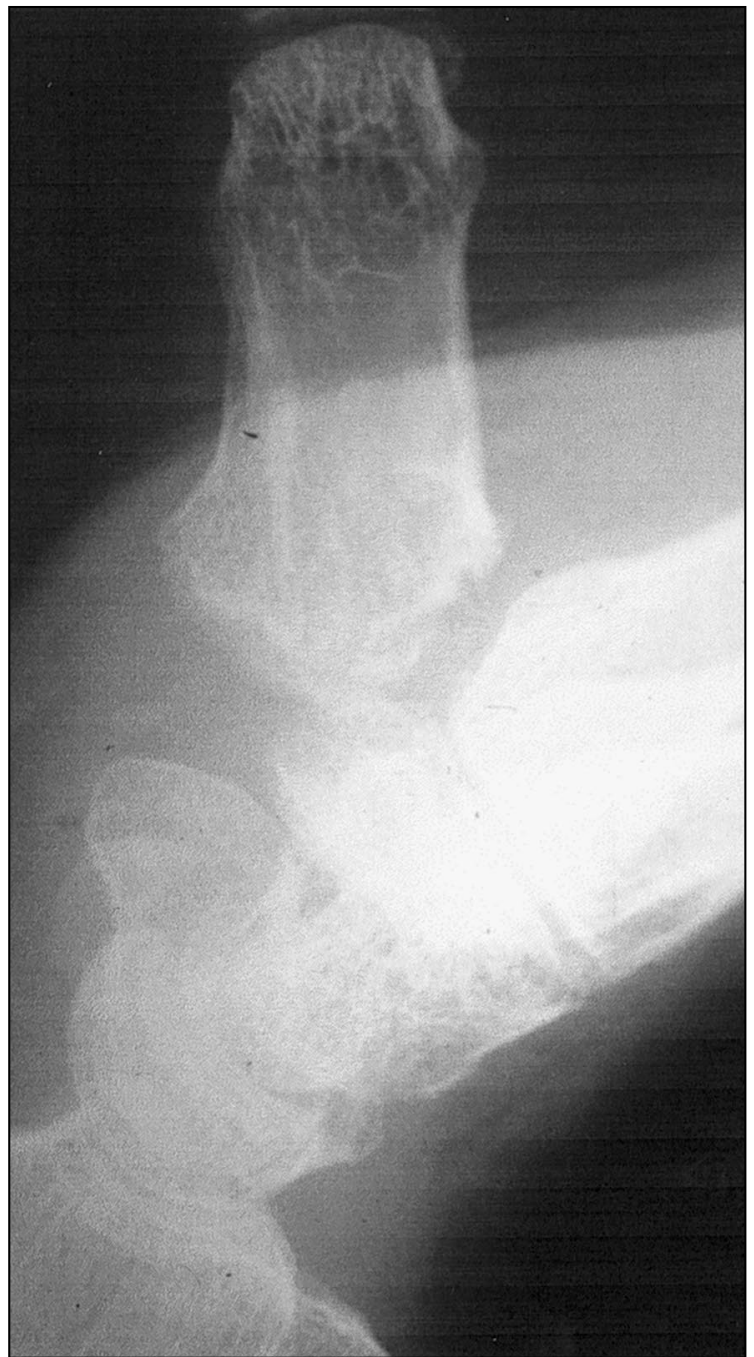

Figura 4. Resultado radiológico de Rx. Figura 3.

\section{DESVENTAJAS DE LA ARTRODESIS TMC}

Hay múltiples desventajas en la realización de la artrodesis.

1. Predisposición de aumentar la artritis o artrosis en las articulaciones adyacentes por el movimiento supletorio.

2. Significante limitación del movimiento articular y del pulgar.

3. Limitada capacidad de abrir totalmente el arco transverso de la mano.

4. Necesidad de larga inmovilización.

5. Hiperextensión compensatoria de la articulación MCF.

6. Posibilidades de pseudoartrosis, la literatura la describe entre el $15 \%$, el $17,4 \%$ y el $29,4 \%$ (1). 


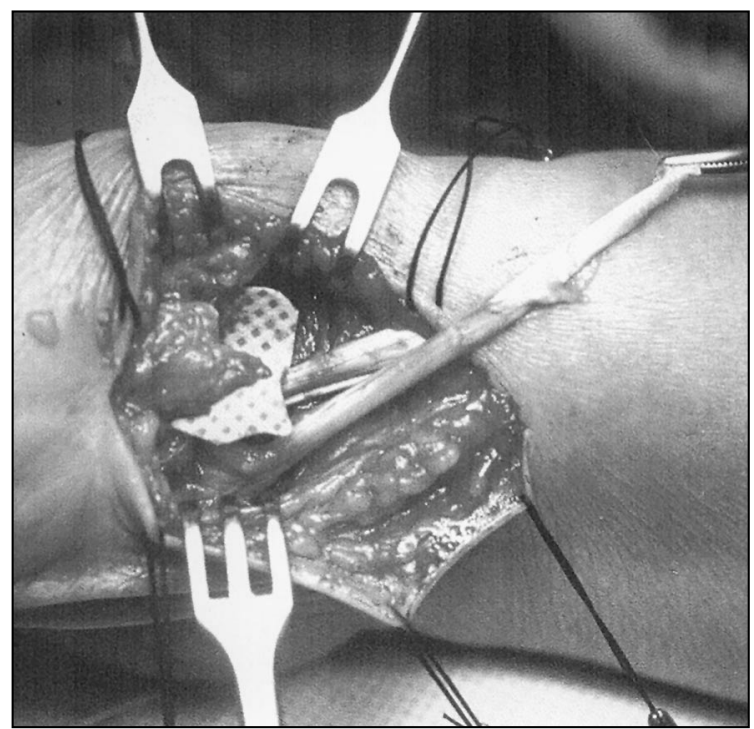

Figura 5. Parte de la técnica quirúrgica. Individualización y sección proximal del APL (Digástrico), que se inserta en masa tenar, para reconstruir el ligamento palmar oblicuo. Introduciéndolo en la base del metacarpiano y luego directamente debajo de la arteria radial hasta unirlo al ECRL y ECRB.

7. Necrosis del trapecio por su precaria irrigación (8).

La indicación precisa es en pacientes jóvenes deportistas o trabajadores manuales, y en pacientes con LUPUS o enfermedad de EHLER-DANLOS (7) y en secuelas severas de parálisis.

\section{EVOLUCIÓN Y TÉCNICA QUIRÚRGICA DE LA ARTROPLASTIA TMC}

El uso de la artroplastia por interposición fue realizada por primera vez por Gervis (9), en 1949, posteriormente han sido descritos otros métodos de artroplastia $(4,9,10)$ con aportes que algunos no han dado el resultado deseado y otros han permanecido vigentes.

Nosotros hemos usado varios métodos. El implante de silicón (10), la anchoa (9), combinaciones de ellos e inclusive la osteotomía de Wilson (13), indicada en el comienzo de la rizoartrosis, no cuando las superficies articulares están comprometidas.

En 1987 comenzamos a usar la artroplastia estabilizadora por interposición con la técnica de Zancolli (14), que utiliza uno de los tendones del abductor largo del pulgar (digástrico)

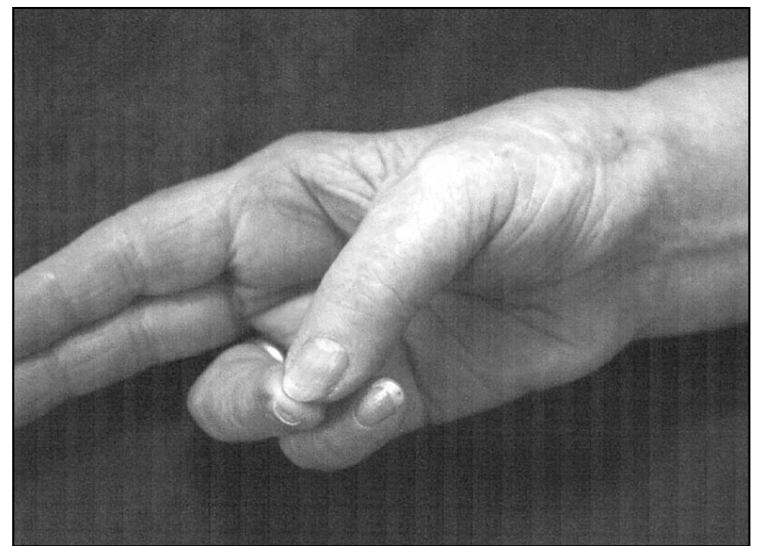

Figura 6. Resultado de un caso con excelente oposición: abducción, adducción y pinzas sin dolor.

introducido en un orificio realizado en la base del primer metacarpiano, para reconstruir el ligamento que le da la estabilidad a la artroplastia, utilizando la cápsula como él lo describió para interponer entre el escafoides y el primer metacarpiano, suturada alrededor del nuevo ligamento reconstruido con el tendón del APL, pero modificamos su técnica porque no lo insertamos en el flexor carpis radialis, lo insertamos al ECRL y ECRB, modificando también así la técnica de Thompson (11), que lo introduce dentro de la base del segundo metacarpiano, después de pasarlo dentro del primero y luego lo inserta en los extensores de la muñeca, como lo hacemos nosotros.

La reconstrucción del ligamento le da suficiente estabilidad y unida a la cápsula mantiene el espacio entre el escafoides y la base del primer metacarpiano. El procedimiento lo realizamos como una cirugía ambulatoria, ello disminuye los costos y la incomodidad del paciente. A la tercera semana se retira el alambre de Kirchsner colocado en el acto quirúrgico y una férula de yeso y comienza el plan formal de rehabilitación, pero se debe usar una férula estática interrumpidamente por 6 semanas para que se realicen las actividades de la vida diaria. Sin pasarlo a través del metacarpiano, sino debajo de la arteria radial.

\section{RESULTADOS}

El programa de rehabilitación es muy importante para obtener excelentes y buenos resulta- 
dos, mayor tiempo de artrodesis ameritó mayor tiempo de rehabilitación (16). Ésta duró de 8 a 17 semanas, en un período de 10 .

El control fue de 2 a 58 meses con promedio de 13, de los 8 pacientes, 2 apenas asistieron a rehabilitación y controles por 4 y 6 semanas pero no fueron controlados hasta el alta.

Rutinariamente se hicieron controles radiológicos en las 6 semanas del postoperatorio y al darle de alta no se apreciaron sublujaciones ni inestabilidad radiológica ni clínica, y el espacio entre el escafoides y la base del primer metacarpiano osciló entre 1 a $5 \mathrm{~mm}$, los mejores resultados tenían un espacio de $3 \mathrm{~mm}$.

De los 6 pacientes controlados hasta el alta, 3 artrodesados secuela de trauma y 3 por rizoartrosis fueron evaluados por el senior autor según el protocolo de EATON (4).

Resultado excelente: número de pacientes: 03

No dolor, fuerza de pinza $90 \%$ del pulgar contralateral, pulgar toca pulpejo del meñique, extensión y no, abducción completa, volvieron a su trabajo original.

Resultado bueno: número de pacientes: 02

Dolor muy ocasional después de prolongadas actividades de fuerza, pulgar toca pulpejo del meñique, extensión-abducción del pulgar normal, fuerza de pinza $70 \%$ del pulgar contralateral.

Resultado regular: número de pacientes: 01

Dolor con pinzas fuertes, fuerza: 50 a $70 \%$ del pulgar contralateral, pulgar a $2 \mathrm{cms}$ del pulpejo del índice, faltan $20^{\circ}$ para extensión-abducción del pulgar.

\section{Resultado malo: no pacientes}

Dolor muy frecuente, primer metacarpiano adductus, hiperextensión de la MCF.

Todos los pacientes manifestaron que volverían a intervenirse y estaban satisfechos no só- lo por no tener dolor, sino también por haber mejorado su funcionalidad.

\section{COMPLICACIONES}

Tuvimos 3 complicaciones, 2 neuropraxias por las incisiones y las múltiples ramificaciones del nervio radial sensitivo en la herida operatoria, y 1 infección superficial curada en 7 días.

\section{DISCUSIÓN}

Convertir una artrodesis de la trapecio-metacarpiana a artroplastia no es una técnica muy común. De la bibliografía manejada Uriburi (12) de 227 casos operados, 10 fueron de salvamento, o sea, reintervenciones, y 4 de ellos eran artrodesis que las convirtió en artroplastias. Podemos decir que éste es un procedimiento de rescate, el motivo más frecuente de ese rescate es la incapacidad producida; en 3 de nuestros pacientes la artrodesis no había quedado en una posición funcional, ello no es simple (1). La artrodesis elimina casi totalmente la circunducción de la articulación dando como resultado quizás mayor fuerza de la pinza que no todos los pacientes necesitan, pero también disminuye el arco de movilidad para la oposición y la abducción.

Consideramos que la artrodesis de la articulación TMC da como resultado estabilidad articular y elimina el dolor, pero nuestra experiencia también indica que disminuye la movilidad del pulgar e influye negativamente en sus diferentes pinzas, por lo que la artroplastia estabilizadora o por interposición le disminuye notablemente la incapacidad, concluyendo que la artrodesis de la TMC tiene indicaciones muy precisas y excepcionales. 


\section{BIBLIOGRAFÍA}

1. Bamberger, H. B.; Stern, P. L.; Kiefhaber, T. R.; McDonough, J. J.; Cantor, R. M.: Trapezio Metacarpal joint arthrodesis. A funciontal evaluation. J. Hand Surg. 1992; 17A; 605-611.

2. Bettinger, P. C.; Snutz, W. P. Linscheid, R. L.; Cooney, W. P.; Kai-nan, A.: Material properties of Trapezial and Trapeziometacarpal ligaments. J. Hand Surg. 2000; 25A; 10851095.

3. De Santolo, A.; Mora, J. G.: Rizoartrosis; Actualización de su tratamiento. Revista de la Facultad de Medicina UCV. 1982; 5: 229-243.

4. Eaton, R. G.; Eaton, S. Z.; Littler, J. W.: Tendon interposition arthroplasty for degenerative arthritis of the trazio-metacarpal joint of the Tuhmb. J. Hand Surg. 1985; 10 A: 645-653.

5. Gervis, W. H.: Excision of the trapezium for osteoarthritis of the trapezio-metacarpal joint.
J. Bone Joint Surg. 1949; 31: 537-539.

6. Gettle, K. A.; Janson, J. R.; Cannon, N. W.: Basilar joint pain, conservative and post-operative management. The Indiana Hand Center. Bulletin 1995; 5: 10-12.

7. Roberts, R. A.; Jabaley, M. E., Nick, T. Q.: Results following trapeziometacarpal Arthroplasty of thumb. J. Hand therapy. 2001; 14: 202-207.

8. Sennwald, G. R.; Seamuller, G.: The value of scaphotrapeziotrapezoid arthrodesis combined with «de la Caffiniere» arthroplasty for the treatmen of pentrapezial osteoathritis. J. Hand Surg. 1993; 18 B: 527-539.

9. Robinson, D.; Aghasi, M.; Halperin, N.: Abductor pollicis Longus tendon arthroplasty of the trapezio-metacarpal joint; Surgical technique and results. J. Hand Surg. 1991; 16 A: 504509.
10. Swanson, A. B.: Disabling arthritis at the base of the thumb, treatment by resection of the trapezium and flexible (silicone) implant arthroplasty. J. Bone Joint Surg. 1972; 54 A: 456471.

11. Thompson, J. S.: Suspensionplasty technique. Atlas of Hand Clinics 1997; 2: 101-120.

12. Uriburi, I. J. F.; Olazábal, A. E.; Ciaffim: Trapezio-metacarpal osteoarthritis: Surgical tecnique and results of «stabilized resection - arthroplasty». J. Hand Surg. 1992; 17 A: 598604.

13. Wilson, J. N.; Bosley, C. J.: Osteotomy in the treatment of osteoarthritis of the first carpometacarpal joint. J. Bone Joint Surg. 1983; 65 B: 179-181.

14. Zancolli, E. A.; Zancolli, E. R.: Osteoarthritis of the trapeziometacarpal joint. The Indiana Hand Center. Bulletin, 1995; 5: 4-9. 